\title{
Peculiar photoinduced properties of water-soluble, early lanthanide(III) porphyrins
}

\author{
Muhammad Imran, Csanád Szentgyörgyi, Gábor Eller, Zsolt Valicsek* and Ottó Horváth \\ Department of General and Inorganic Chemistry, Institute of Chemistry, Faculty of Engineering, \\ University of Pannonia, P.O.B. 158, Veszprém H-8201, Hungary
}

\begin{abstract}
Water-soluble, early lanthanide(III) mono- and bisporphyrin complexes possess very similar UV-Vis absorption, photophysical and photochemical properties, as a consequence of a special type of aggregation, through the peripheral substituents. In the absence of the bidentate, O-donor acetate ligand, bisporphyrin can form too, which has slightly redshifted and broadened absorption bands, compared to those of the monoporphyrin. Also the bisporphyrin displays a blueshifted and less intense fluorescence, related to the free-base porphyrin. The formation of complexes and the transformation between the mono- and bisporphyrins are very slow reactions in dark at room temperature. These reactions are accelerated by the photolysis of the system; which are considerable by-processes of the photoredox degradation. Depending on the wavelength of irradiation, two types of photoproducts can appear: during the photolysis at the Soret-band, a radical type intermediate can be observed, which disappears in dark. However, during the irradiation at the Q-bands, a new photoproduct appears, which is stable in dark and undetectable in the case of post-transition metal ions' out-of-plane porphyrin complexes.
\end{abstract}

Keywords:

out-of-plane metalloporphyrins, early lanthanide(III) ions, tail-to-tail oligomerization, LMCT, charge separation, water cleavage.

\footnotetext{
* Corresponding author. Tel.: +36 (88) 624 431; fax: +36 (88) 624548 (Z. Valicsek).

E-mail addresses: valicsek@vegic.uni-pannon.hu (Z. Valicsek), muhammadimranum@yahoo.com (M. Imran), fehron@gmail.com (C. Szentgyörgyi), gaboreller@gmail.com (G. Eller), otto@ vegic.unipannon.hu (O. Horváth).
} 
Metal ions can form in-plane metalloporphyrins, fitting into the cavity of the porphyrin ring, or several of them are located out of the ligand plane, resulting in out-of-plane (OOP or sitting-atop=SAT) complexes with dome-distorted structure, thermodynamic instability, kinetic lability, typical photophysical features, and photochemical reactivity $[1,2]$. Besides, the OOP position promotes the formation of bis- or oligoporphyrins, so called sandwich complexes, mainly if the metal center is susceptible to higher coordination numbers (Fig. 1) [3-6]. Lanthanide(III) complexes are the model compounds of metallo-oligoporphyrins, in which the $\pi-\pi$ interaction depends on the distance between the macrocycles, strongly influenced by the size of the metal center [7-9]. Therefore, lanthanide(III) ions offer good opportunities to examine this dependence due to the fine tuning of the out-of-plane distances, utilizing the wellknown lanthanide contraction: the radii of $\mathrm{Ln}^{3+}$ ions monotonously decrease from $116 \mathrm{pm}$ to 98 pm (at coordination number 8) with the increase of atomic numbers within the lanthanide series (Fig. 2) [10]. As a consequence of their strong susceptibility to higher coordination numbers, the investigation of their monoporphyrins is complicated: another multidentate, but O-donor ligand must be applied to hinder the coordination of the second porphyrin to the metal ion [1116]. From optical and photophysical aspects, porphyrins are able to efficiently sensitize the near-infrared luminescence of lanthanide ions, which can be widely applied, e.g. in optical telecommunication, spectral conversion of solar energy, and biomedical optical imaging [1116]. Furthermore, lanthanide(III) porphyrins may be useful in the photocatalytic cleavage of water because the metal center, reduced due to the photoinduced charge transfer from ligand to metal (LMCT) $[1,2,17,18]$, has a negative redox potential large enough to produce hydrogen (except Eu ${ }^{3+}$, Fig. 2) [9].

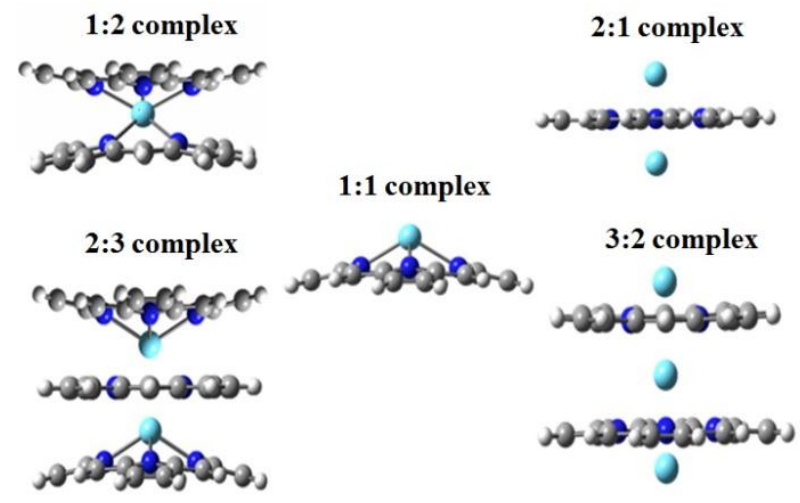

Fig. 1. Structure of the out-of-plane complexes with various metal:porphin compositions.

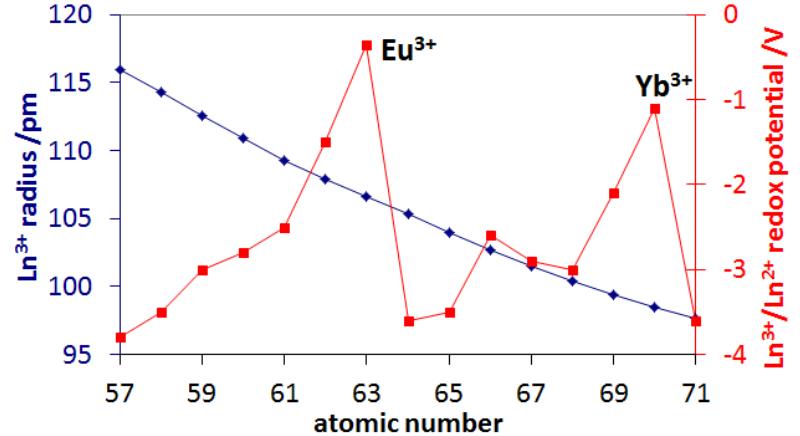

Fig. 2. Contraction and redox potentials of lanthanide(III) ions.

In this work, we studied the photophysical and primary photochemical properties of the complexes between a water-soluble, anionic porphyrin, the 5,10,15,20-tetrakis(4sulfonatophenyl)porphyrin anion $\left(\mathrm{H}_{2} \mathrm{TSPP}^{4-}\right.$, abbreviated as $\mathrm{H}_{2} \mathrm{P}^{4-}$ ) and early lanthanide(III) ions ( $\mathrm{Ln}=\mathrm{La}, \mathrm{Ce}, \mathrm{Nd}, \mathrm{Sm})$. Lanthanide(III) ions are hard Lewis acids, due to the classification of Pearson, therefore, their insertion into the coordination cavity of the tetradentate, N-donor porphyrin ligand is a slow and complicated process in aqueous solution, originating partly from the strong bond of the solvent water molecules to the metal ions [13, 19-22]. This kinetic barrier is vanquishable, e.g. by heating: in our case to $\sim 60^{\circ} \mathrm{C}$ [22]. The presence of bidentate, O-donor acetate ion enhances the coordination of the first porphyrin ligand, due to its trans effect, but it hinders the connection of a further porphyrin, i.e. the formation of bisporphyrins, because it remains on the lanthanide(III) ion in axial position [9, 22]. Insertion of these larger metal ions 
into the porphyrin cavity can be spectrophotometrically followed due to the redshifts of UVVis, intraligand $\pi \pi^{*}$ absorption bands (Fig. 3), similarly to other typical OOP complexes, which display "common" absorption properties [23]. In the absence of the bidentate, O-donor ligand, bisporphyrin $\left(\mathrm{Ln}_{3} \mathrm{P}_{2}{ }^{3-}\right)$ can form too, which has slightly redshifted and broadened absorption bands, compared to those of the monoporphyrin.

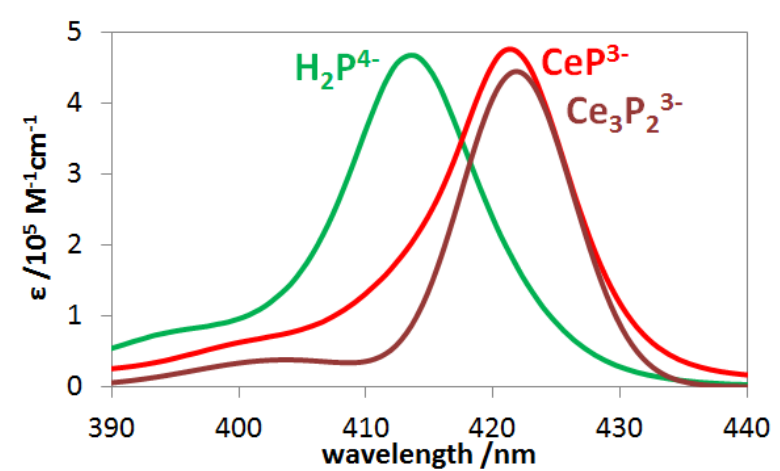

Fig. 3. Molar absorption spectra of cerium(III) mono- and bisporphyrin as well as the corresponding free base in the Soretrange.

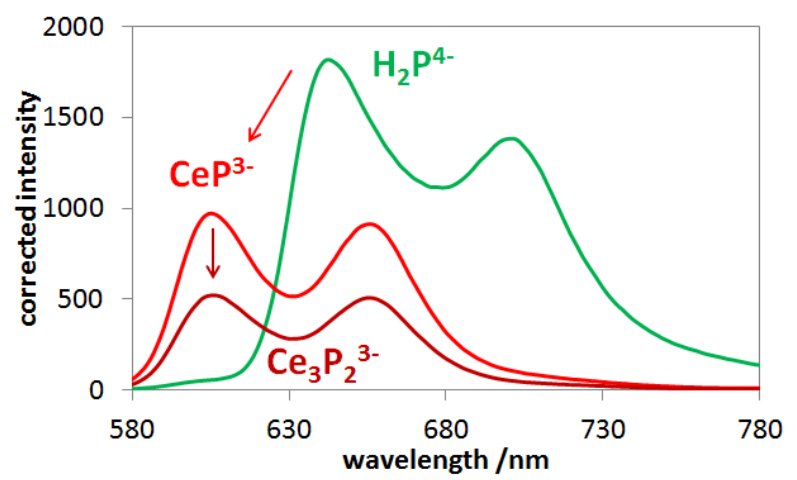

Fig. 4. Singlet-1 fluorescence spectra of cerium(III) mono- and bisporphyrin as well as the corresponding free base.

These bisporphyrins display a blueshifted and less intense singlet-1 fluorescence, related to the free-base porphyrin (Fig. 4). While the lifetimes for mono- and bisporphyrin complexes are almost equal $(\sim 2 \mathrm{~ns})$, only the fluoresence quantum yield decreases slightly because the formation of bisporphyrin results only in the deceleration of radiative decay, as a consequence of a special type of aggregation, probably through the peripheral, sulfonato substituents $[16,22$, $24,25]$ (tail-to-tail or perpendicular head-to-tail dimerization) without strong $\pi$ - $\pi$ interactions between the macrocycles $[22,23]$. If these interactions were stronger, the absorption bands should show much larger redshifts and hyperchromicities, and the fluorescence should be much weaker, nearly disappear. This was manifested in the case of the (parallel) head-to-tail dimer of the protonated porphyrin, $\left(\mathrm{H}_{4} \mathrm{TSPP}^{2-}\right)_{2}$ [26], and of the bisporphyrins of mercury(II) ion: (parallel) head-to-tail $\mathrm{Hg}_{2}{ }_{2}$ (TSPP $)_{2}{ }^{8-}$ and typical head-to-head $\mathrm{Hg}_{3}{ }_{3}$ (TSPP $)_{2}{ }^{6-}[5,23]$.

The photoinduced reactions of lanthanide(III) mono- and bisporphyrins are also very similar in both the Soret- (Fig. 5) and the Q-band-photolysis experiments (Fig. 6). Also this phenomenon may confirm this special type of aggregation through the peripheral sulfonato substituents because in the case of post-transition metal ions the differences are much larger, mainly in the range of Q-bands $[5,6]$.
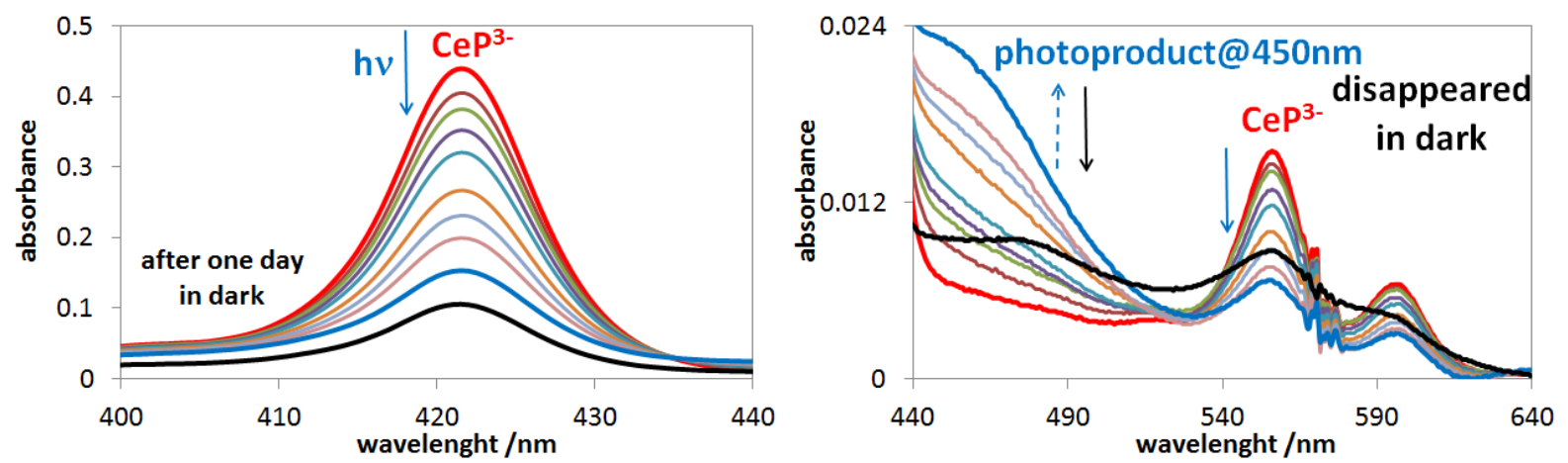

Fig. 5. Spectral changes in the range of Soret- and Q-bands during the photolysis at the Soretmaximum (421 $\mathrm{nm})$. 
The out-of-plane position of the metal center in porphyrin complexes can generally cause two types of photochemical reactions: 1) photoinduced dissociation of the metal ion from the cavity of the ligand (without charge transfers) as a consequence of the lability of the complex; 2) photoredox degradation of the macrocycle initialized by an irreversible LMCT reaction resulting in oxidation of the ligand and reduction of the metal center. The charge of the metal center decreases, and its size increases, thus its charge density falls down, hence the coordinative bonds can easily split. The reduced metal ion can step out from the cavity (Fig. 7), mainly in polar solvent, and can induce further redox reactions, depending on its stability in the given medium (Fig. 2). The oxidized and metal-free porphyrin (cat)ionic radical is a very strong base: it gets immediately protons, forms the free-base radical, which is long-lived and a relatively strong oxidizer, mainly in oxygen-free solution $\left(\tau_{1 / 2}>>1 \mathrm{~ms}, \mathrm{E}_{1 / 2}>1 \mathrm{~V}\right)$ [27]. But it would oxidize water to oxygen only at higher $\mathrm{pH}$, therefore a slightly stronger reducer, for example water-soluble alcohols or aldehydes, are required, from which useful byproducts can be formed in photocatalytic hydrogen production. In the absence of reducer (without regeneration, cyclization), the primary photochemical processes take place: in an overall fourelectron oxidation, ring-opening reaction, a dioxo-tetrapyrrole derivative (bilindione) can form (Fig. 7) [1, 2, 27]. This ring-opening process can be spectrophotometrically followed, due to the disappearance of the Soret-band, and a radical type intermediate can be observed at $450 \mathrm{~nm}$, which disappears in dark (Fig. 5).

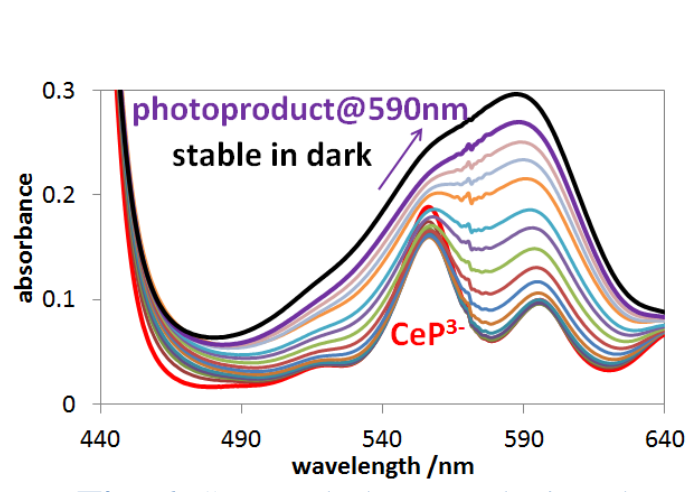

Fig. 6. Spectral changes during the photolysis at the Q-maximum $(555 \mathrm{~nm})$.

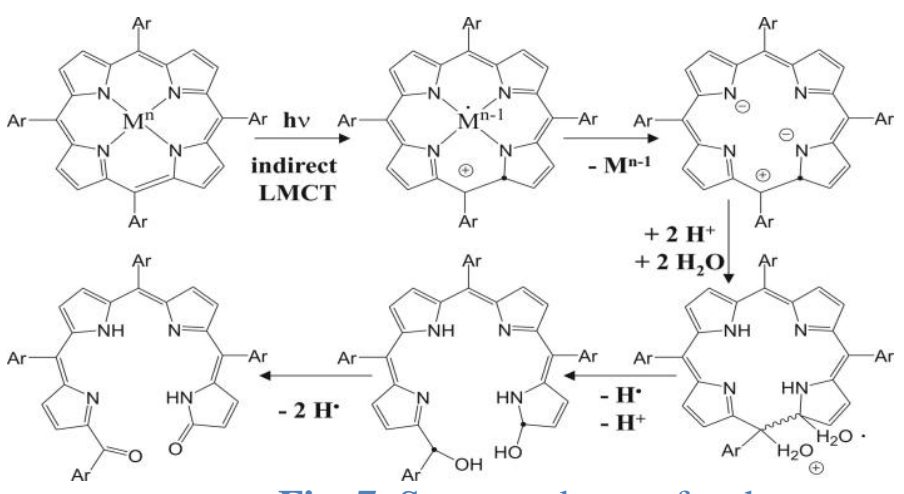

Fig. 7. Suggested steps for the photodegradation of OOP metalloporphyrins [2].

However, during the photolysis of early lanthanide(III) porphyrins at the maximum of the Q-bands $(\sim 555 \mathrm{~nm})$, a new photoproduct appears, which is stable in dark (Fig. 6) and undetectable in the case of post-transition metal ions' typical OOP complexes [1,2]. The Soretband disappeared as a consequence of the ring-opening reaction; hence, the absorption band at $590 \mathrm{~nm}$ may be assigned to a complex between lanthanide(III) and this open-chain, dioxotetrapyrrole derivative (Fig. 7), owing to the high affinity of these metal ions to the oxo-groups.

In the absence of acetate, the lanthanide(III) mono- and bisporphyrins are in equilibrium, moreover free-base ligands may also remain; hence, during the photolyses, they are simultaneously excited. Owing to a deeper evaluation of spectral changes, also photoinduced transformation between the mono- and bisporphyrin complexes can be identified, beside the photoredox degradations to the mentioned, open-chain photoproducts and dissociation to the free-base porphyrin. The equilibria between the complexes are varied by excitation, i.e. the significant parts of the overall photochemical quantum yields originate from these photoinduced transformations of complexes to each other in this photostationary state [9]. Light 
accelerates these reactions by orders of magnitude, compared to their very low rate in dark at room temperature [22].

For the determination of photochemical quantum yields, the widely-used initial slope method is inappropriate because the initial absorbance also decreases considerably (Fig. 5), furthermore, other absorbing species may appear during the photolysis (Fig. 5 and Fig. 6). In such a case the so-called integral fitting method and the evaluation method based on the concentration distribution of the absorbing species may be suitable to understand the photochemical mechanism and to determine the quantum yields for each type of photoinduced reactions. First, we studied the effect of ionic strength: the overall photochemical quantum yield displayed a quasi-linear dependence on that as a consequence of the enhanced possibility of the photoinduced charge separation after LMCT. The effect of the porphyrin concentration was also investigated: the overall photochemical quantum yields monotonously increased with the concentration. This suggests that the excited complex molecules can undergo bimolecular, thermal (redox) reaction with the species in ground state [9].

More profound studies about these photoinduced reactions are in progress for all lanthanide(III) ions, to find tendencies between the photoactivities and the out-of-plane distances.

\section{Acknowledgments}

This work was supported by the Hungarian Scientific Research Fund (NN107310), the Hungarian Government and the European Union, with the co-funding of the European Social Fund (TÁMOP-4.2.2.A-11/1/KONV-2012-0071), and the Austrian-Hungarian Action Foundation (86öu3).

\section{References}

1. O. Horváth, R. Huszánk, Z. Valicsek, G. Lendvay, Photophysics and photochemistry of kinetically labile, water-soluble porphyrin complexes, Coord. Chem. Rev. 250 (2006) 1792-1803.

2. O. Horváth, Z. Valicsek, G. Harrach, G. Lendvay, M.A. Fodor, Spectroscopic and photochemical properties of water-soluble metalloporphyrins of distorted structure, Coord. Chem. Rev. 256 (2012) 1531-1545.

3. J.W. Buchler, A. Decian, J. Fischer, M. Kihnbotulinski, H. Paulus, R. Weiss, Metal-complexes with tetrapyrrole ligands 40. Cerium(IV) bis(octaethylporphyrinate) and dicerium(III) tris(octaethylporphyrinate) Parents of a new family of lanthanoid double-decker and triple-decker molecules, J. Am. Chem. Soc. 108 (1986) 3652-3659.

4. G. Ricciardi, A. Rosa, E.J. Baerends, S.A.J. van Gisbergen, Electronic Structure, Chemical Bond, and Optical Spectra of Metal Bis(porphyrin) Complexes: A DFT/TDDFT Study of the Bis(porphyrin)M(IV) (M ) Zr, Ce, Th) Series, J. Am. Chem. Soc. 124 (2002) 12319-12334.

5. Z. Valicsek, G. Lendvay, O. Horváth, Equilibrium, photophysical, photochemical and quantum chemical examination of anionic mercury(II) mono- and bisporphyrins, J. Phys. Chem. B. 112 (2008) 14509-14524.

6. Z. Valicsek, G. Lendvay, O. Horváth, Equilibrium, photophysical, photochemical and quantum chemical examination of anionic mercury(I) porphyrins, J. Porph. Phthal. 13 (2009) 910-926.

7. J. Jiang, M. Bao, L. Rintoul, D.P. Arnold, Vibrational spectroscopy of phthalocyanine and naphthalocyanine in sandwich-type (na)phthalocyaninato and porphyrinato rare earth complexes, Coord. Chem. Rev. 250 (2006) 424-448.

8. X. Zhang, Y. Chen, A sandwich mixed (phthalocyaninato) (porphyrinato) europium triple-decker: Balancedmobility, ambipolar organic thin-film transistor, Inorg. Chem. Commun. 39 (2014) 79-82.

9. Z. Valicsek, G. Eller, O. Horváth, Equilibrium, photophysical and photochemical examination of anionic lanthanum(III) mono- and bisporphyrins: the effects of the out-of-plane structure, Dalton Trans. 41 (2012) 13120-13131.

10. R.D. Shannon, Revised Effective Ionic Radii and Systematic Studies of Interatomic Distances in Halides and Chalcogenides, Acta Cryst. A32 (1976) 751-767.

11. G. Knör, A. Strasser, Coexisting intraligand fluorescence and phosphorescence of hafnium(IV) and thorium(IV) porphyrin complexes in solution, Inorg. Chem. Commun. 5 (2002) 993-995.

12. G. Knör, A. Strasser, Enhanced photoreactivity of zirconium(IV) and hafnium(IV) porphyrin complexes promoted by water molecules, Inorg. Chem. Commun. 8 (2005) 471-473. 
13. H. He, A.G. Sykes, D. Galipeau, S. Weng Ng, M. Ropp, Crystallography and photoluminescence properties of b-diketonate monoporphyrinate ytterbium(III) complexes, Inorg. Chem. Commun. 11 (2008) 1051-1053.

14. H. He, A.G. Sykes, Facile preparation of neutral monoporphyrinate lanthanide complexes with strong nearinfrared emission, Inorg. Chem. Commun. 11 (2008) 1304-1307.

15. H. He, Near-infrared emitting lanthanide complexes of porphyrin and BODIPY dyes, Coord. Chem. Rev. 273 (2014) 87-99.

16. V. Bulach, F. Sguerra, M.W. Hosseini, Porphyrin lanthanide complexes for NIR emission, Coord. Chem. Rev. 256 (2012) 1468-1478.

17. F.W. Oliver, C. Thomas, E. Hoffman, D. Hill, T.P.G. Sutter, P. Hambright, S. Haye, A.N. Thorpe, N. Quoc, A. Harriman, P. Neta, S. Mosseri, Mössbauer, magnetic susceptibility, radiolytic and photochemical studies of europium and lutetium porphyrins, Inorg. Chim. Acta 186 (1991) 119-124.

18. H. Kunkely, A. Vogler, Photodemetalation of silver(II) tetraphenylporphyrin, Inorg. Chem. Commun. 10 (2007) 479-481.

19. Y. Yamaguchi, M. Nomura, H. Wakita, H. Ohtaki, An extended x-ray absorption fine structure study of aqueous rare earth perchlorate solutions in liquid and glassy states, J. Chem. Phys. 89 (1988) 5153-5159.

20. L. Helm, A.E. Merbach, Water exchange on metal ions: experiments and simulations, Coord. Chem. Rev. 187 (1999) 151-181.

21. S. Haye, P. Hambright, Acid solvolysis kinetics of lanthanide porphyrins, J.C.S. Chem. Commun. (1988) 666668.

22. M.P. Kiss, M. Imran, C. Szentgyörgyi, Z. Valicsek, O. Horváth, Peculiarities of the reactions between early lanthanide(III) ions and an anionic porphyrin, Inorg. Chem. Commun. 48 (2014) 22-25.

23. Z. Valicsek, O. Horváth, Application of the electronic spectra of porphyrins for analytical purposes: the effects of metal ions and structural distortions, Microchem. J. 107 (2013) 47-62.

24. S. Lipstman, S. Muniappan, S. George, I. Goldberg, Framework coordination polymers of tetra(4carboxyphenyl)porphyrin and lanthanide ions in crystalline solids, Dalton Trans. 30 (2007) 3273-3281.

25. M. La Deda, M. Ghedini, I. Aiello, I. De Franco, Zinc porphyrin with phenoxy-bridged pentacoordinate bis (8hydroxyquinaldinate)gallium lateral pendants: synthesis and photophysical characterization, Inorg. Chem. Commun. 7 (2004) 1273-1276.

26. D.L. Akins, S. Özcelik, H.R. Zhu, C. Guo, Fluorescence decay kinetics and structure of aggregated tetrakis(psulfonatophenyl)porphyrin, J. Phys. Chem. 100 (1996) 14390-14396.

27. P. Neta, M. C. Richoux, A. Harriman, L. R. Milgrom, Resonance stabilisation of zinc porphyrin $\pi$-radical cations, J.C.S. Faraday Trans. II. 82 (1986) 209-217. 